\title{
COMMUNICATION-INDUCED DISTURBANCES IN HAPTIC TELEPRESENCE SYSTEMS
}

\author{
M. Kuschel and S. Hirche and M. Buss
}

\author{
Institute of Automatic Control Engineering \\ Technische Universität München \\ D-80290 München, Germany \\ www.Isr.ei.tum.de
}

\{Martin.Kuschel, Hirche\}@tum.de, M.Buss@ieee.org

\begin{abstract}
In force-reflecting telepresence, communication delays can lead to instability. A common approach to passivate the system disturbed by constant delays is to apply the wave transformation. However, this leads to the effect of wave reflections that decrease performance. This paper provides a detailed description of wave reflections as arising in haptic telepresence systems. The influence on the perception of the remote environment is analyzed and validated experimentally.
\end{abstract}

Keywords: teleoperation, communication networks, delay, scattering problems

\section{INTRODUCTION}

Telepresence enables a human operator to perceive and manipulate a remote environment. A telepresence system consists of three components. The human system interface (HSI) is handled by the human operator. He/She commands the teleoperator to perform actions in a remote environment. HSI and teleoperator exchange command and feedback signals over a communication line. To achieve a holistic remote immersion, multiple modes of human perception are addressed including haptic, visual, and auditory senses. The level of the immersion is called the transparency of the telepresence system. Applications for telepresence are telesurgery, teleassembly and telerescue systems, see Fig. 1 for an illustration.

This paper focuses on force-reflecting (i.e. haptic) telepresence with constant time delays. Forcereflecting telepresence systems have severe stability problems when executed in presence of time delays caused by the communication line. The most eligible approach to analyze and design haptic telepresence systems with time delay is the

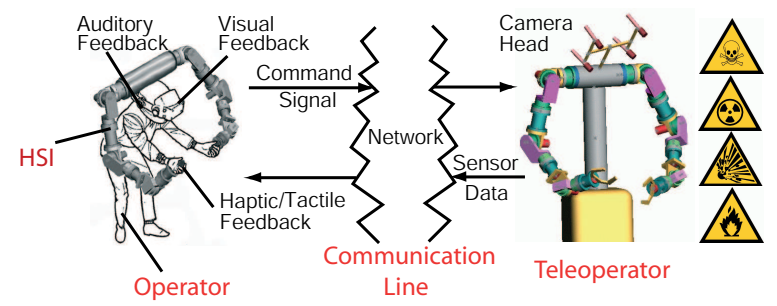

Fig. 1. Multimodal Telepresence

passivity concept which is applied here. In order to passivate the communication line the wave transformation is applied. Wave variables were introduced in (Anderson and Spong, 1989) and extended in (Niemeyer, 1996). This transformation renders the communication line passive to arbitrary constant delays. As a result the dynamics of the communication system equal the dynamics of a lossless electrical transmission line. The dynamics are described by the impedance, the ratio of effort and flow, hence in a mechanical context by the ratio of force and velocity. If the impedance of the remote environment is not matched to the characteristic impedance of the communication line, 
wave reflections, a phenomenon well known from electrodynamics, are excited. Wave reflection decrease the transparency. So far different strategies have been proposed to improve the performance of the telepresence system by impedance matching (Niemeyer and Slotine, 1997) and (Hirche and Buss, 2003). However, these strategies may not completely avoid wave reflections. The influence of wave reflections on transparency has not yet been investigated qualitatively. This paper provides an analysis of wave reflections as they influence the telepresence system, and the perception of the remote environment. Experimental validation is conducted with two-degree-of-freedom SCARArobots.

The remainder is arranged as follows: Section 2 provides with the theoretical background of timedelayed haptic telepresence, including passivity theory, wave transformation and impedance matching. The analysis of wave reflections - the main part - is given in section 3 . In section 4 and 5 simulation as well as experiments are presented.

\section{BACKGROUND}

\subsection{Passivity}

Passivity is an energy based concept well suited to sufficiently guarantee stable performance of complex distributed systems. To apply the notion of passivity the complete system is divided into subsystems. If these subsystems are passive and connected in an appropriate way, overall stability can be ensured for the whole system. A passive system absorbs more energy than it generates, i.e. the power entering a system is larger than the change of the energy being stored inside. Power input is defined as scalar product between the input vector $\dot{\mathbf{x}}$ and the output vector $\mathbf{F}$,

$$
P_{\text {in }}=\dot{\mathbf{x}}^{T} \mathbf{F} .
$$

It is defined to be positive when entering the system and does not necessarily correspond to any physical power. A sufficient condition for passivity is

$$
\int_{0}^{t} P_{\text {in }}(\tau) d \tau \geq-E_{\text {store }}, \quad \forall t \geq 0,
$$

where $E_{\text {store }}$ represents the energy that is stored in the system. For systems with no initially stored energy this condition becomes

$$
\int_{0}^{t} P_{i n}(\tau) d \tau \geq 0, \quad \forall t \geq 0 .
$$

For linear SISO systems the transfer function must be positive real. This is equivalent to the condition

$$
|\angle G(j \omega)| \leq \frac{\pi}{2}, \quad \forall \omega \geq 0 .
$$

For strict passivity the inequality holds. A test if a transfer function might be (strict) positive real can be done by means of its coefficients $n_{i}$ and $d_{j}$. For (strict) positive realness they must at least obey

$$
n_{i} \geq 0 \wedge d_{j} \geq 0 \quad i, j \geq 0 .
$$

For strict passivity all coefficients must be greater than zero.

As illustrated in Fig. 2 a telepresence system can be interpreted as a connection of two-ports terminated by one-ports at each end. The complete system is passive if all subsystem, i.e. all connected two-ports and the terminating one-ports are passive themselves.

To obtain a passive telepresence system the oneport on the left consisting of the human operator handling the HSI has to be passive. A trained operator is supposed to assure this. The right one-port consisting of the teleoperator working in the remote environment must be passive as well. This can at least be achieved by an appropriate local velocity controller. The communication twoport affected by constant delays is passivated by deploying the wave transformation.

\subsection{Wave Transformation}

The communication causes a time delay of the submitted signals. The communication two-port with time delay, however, is not passive as shown in (Anderson and Spong, 1989). In order to design a passive and therefore stable telepresence system all its subsystems must be passive. Motivated by a lossless electrical transmission line and based on scattering theory a transformation of the active communication line to a passive one is introduced in (Anderson and Spong, 1989). In (Niemeyer, 1996) this approach is developed further and wave variables are introduced and applied to telepresence systems with velocity control and force reflection. The transformation of velocity and force into wave variables is given by

$$
\begin{aligned}
& u_{h s i}=\frac{b \dot{x}_{h s i}+F_{h s i}}{\sqrt{2 b}}, \quad v_{h s i}=\frac{b \dot{x}_{h s i}-F_{h s i}}{\sqrt{2 b}}, \\
& u_{t e l}=\frac{b \dot{x}_{t e l}+F_{t e l}}{\sqrt{2 b}}, \quad v_{t e l}=\frac{b \dot{x}_{t e l}-F_{t e l}}{\sqrt{2 b}},
\end{aligned}
$$

where $u$ describes the incident wave and $v$ the reflected wave. The parameter $b$ represents the wave impedance. It must be a positive constant or a positive definite matrix and can be chosen arbitrary.

Since this mapping is bijective it is always unique and it is always invertible. The normalization by the root term in equations (6) is done to achieve the identity 


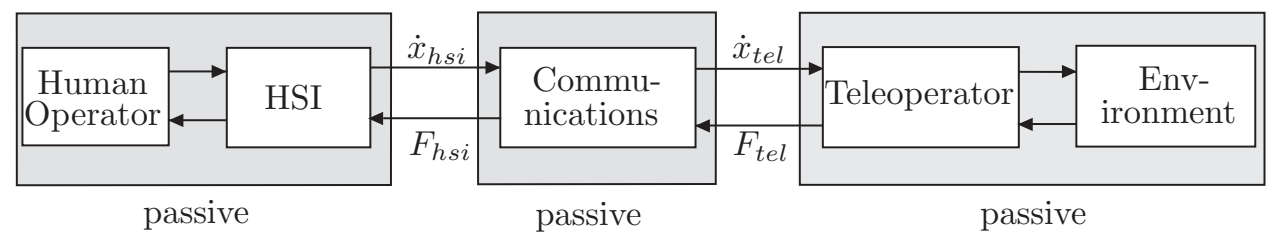

Fig. 2. Passive subsystems assure overall stability

$$
P_{\text {in }}=\dot{\mathbf{x}}^{T} \mathbf{F}=\frac{1}{2} \mathbf{u}^{T} \mathbf{u}-\frac{1}{2} \mathbf{v}^{T} \mathbf{v} .
$$

Passivity can be verified by computing condition (3) in the wave domain. Assuming a constant delay of $T_{1}$ in the forward and $T_{2}$ in the backward path and applying equation (7) yields

$$
\begin{gathered}
\int_{0}^{t} P_{i n}(\tau) d \tau=\int_{0}^{t}\left(\dot{x}_{l}^{T} F_{l}-\dot{x}_{r}^{T} F_{r}\right) d \tau= \\
\frac{1}{2} \int_{t-T_{1}}^{t} u_{h s i}^{2} d \tau+\frac{1}{2} \int_{t-T_{2}}^{t} v_{t e l}^{2} d \tau \geq 0, \quad \forall t \geq 0 .(8)
\end{gathered}
$$

Hence the passivity condition is never violated. Consequently, arbitrary constant time delays are passive in wave domain.

\subsection{Impedance Matching}

The transfer function between force $F$ and velocity $\dot{x}$ is known as mechanical impedance

$$
Z(s)=\frac{F(s)}{s x(s)} .
$$

It describes the dynamical resistance of a mechanic environment analog to an electrical impedance. Since the telepresence system has a velocity/force architecture all subsystems illustrated in Fig. 3 represent impedances like the human operator encountering the impedance $Z^{*}$ as well as the teleoperator encountering the remote environment $Z_{e}$. If the communication two-port is passivated by the wave transformation then wave reflections are generated if the impedances of the communication line and of the remote environment do not match. The effort to avoid wave reflections is called impedance matching. It is well known from electrical transmission lines. In (Niemeyer, 1996) impedance matching is partly reached by an appropriate velocity controller. In (Hirche and Buss, 2003) special impedance matching filters try to adapt the terminating impedance. However, these approaches do not reach impedance matching over all frequencies. Hence wave reflections still have an impact on the transparency.

\subsection{Transparency}

The telepresence system is called transparent if the operator is not able to distinguish between a direct interaction with an environment and the interaction by telepresence. According (Lawrence, $1993)$ the equality of the displayed impedance $Z^{*}$ and the environmental impedance $Z_{e}$ is required for transparency. In the following the impedance is assumed to be approximated by a LTI system $Z(s)=m s^{2}+p s+k$ with inertia $m$, damping coefficient $p$ and stiffness coefficient $k$. If these parameters are equal for the environment and the displayed impedance, then the transparency criteria is assumed to be fulfilled. Under the assumption that the dynamics of the HSI and of the teleoperator have a negligible small influence, the transparency condition can, according to Fig. 3, be rewritten as

$$
Z^{*}\left(k_{h}, m_{h}, p_{h}\right) \stackrel{!}{=} Z_{x}\left(k_{t}, m_{t}, p_{t}\right) .
$$

The indices indicate the independence of the parameters of the operator side (h) and the teleoperator side $(\mathrm{t})$. This measure is used within this paper.

\section{WAVE REFLECTIONS}

Wave reflections contain the energy that is reflected at an impedance discontinuity. They occur if the impedance of the remote system is not matched to the wave impedance $b$ of the communication line. Wave reflections include the information of the reflected force signal but they also carry useless information that deteriorate transparency.

\subsection{Reflection Factor}

Wave variables are linear combinations of velocity and force according to the solution of the wave differential equation with lossless propagation. This leads to an incident wave $u$ and the wave reflections $v$ according to equations (6). The reflection factor $R$ is the transfer function between both waves. In this paper only the reflection factor at the right hand side (Fig. 3 ) of the time delay is considered

$$
R(s)=\frac{v_{r}(s)}{u_{r}(s)} .
$$

The reflection factor of the telepresence system is calculated as

$$
R(s)=\frac{b-Z_{x}(s)}{b+Z_{x}(s)} .
$$




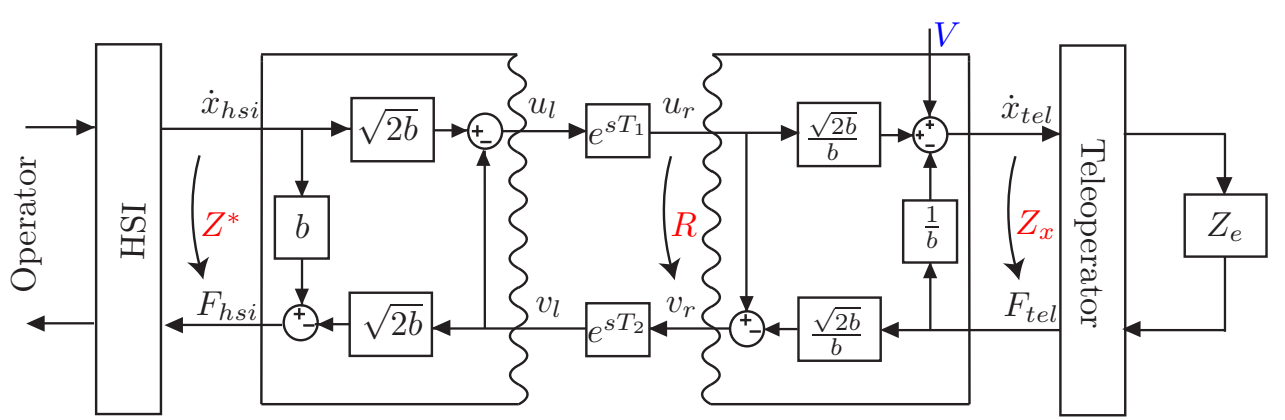

Fig. 3. Structure of the telepresence system. Wavy blocks contain wave transformations.

Hence the dynamic of the wave reflections $v_{r}$ is defined by the characteristic impedance $b$, which can be chosen arbitrary, the impedance $Z_{x}$, terminated by the teleoperator, and the remote environment.

\subsection{Stability}

Stability of a telepresence system afflicted with wave reflections can be determined by analyzing the reflection factor $R(s)$. If the system $Z_{x}$ is not matched to the characteristic impedance $b$ of the communication line, then the remote environment becomes part of a simple closed loop control with unity feedback as sketched in Fig. 4. This

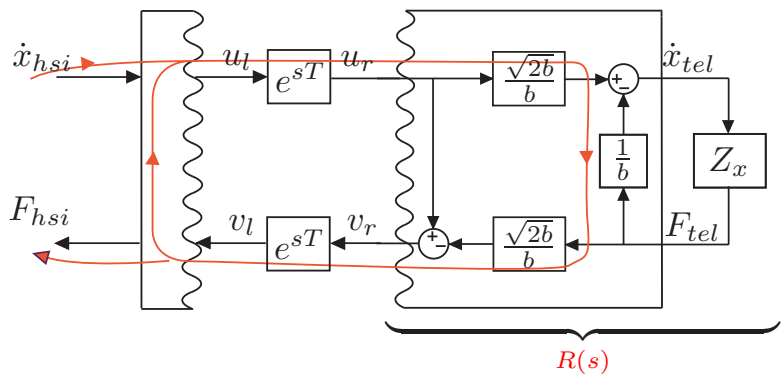

Fig. 4. Reflection factor $R(s)$ determines stability behavior

feedback system is defined by the reflection factor and the time delays in the forward branch. The human operator/HSI that closes the system is not regarded in Fig. 4 because these dynamics are passive, i.e. they do not narrow the stability margin of the total system (Fig. 3). Since the time delays do not cause any amplification but a phase shift greater than $\pi$, the impedance $Z^{*}$ is stable, if the reflection factor $R(s)$ fulfills the small gain theorem.

$$
|R(j \omega)|<1, \quad \forall \omega \geq 0
$$

Inserting equation (12) yields

$$
|R(j \omega)|=\left|\frac{b-Z_{x}(j \omega)}{b+Z_{x}(j \omega)}\right|<1, \quad \forall \omega \geq 0 .
$$

Expressing furthermore $Z_{x}$ by its numerator and denominator

$$
Z_{x}(j \omega)=\frac{\sum_{i} n_{i}(j \omega)^{i}}{\sum_{i} d_{i}(j \omega)^{i}}, \quad i \geq 0,
$$

leads to a stability criterion depending on the coefficients of $Z_{x}$

$$
|R(j \omega)|=\left|\frac{\sum_{i}\left(d_{i} b-n_{i}\right)(j \omega)^{i}}{\sum_{i}\left(d_{i} b+n_{i}\right)(j \omega)^{i}}\right|<1, \quad i \geq 0 .
$$

This inequality holds for

$$
Z_{x}(j \omega): \mathbf{R}^{+} \rightarrow \mathbf{R}^{+} \backslash\{0, \infty\},
$$

According to the passivity test (5) all strictly passive systems meet this condition. This stability result confirms the passivity based design of the telepresence system that has already assured stability. However, applying condition (16) provides one of the contributions of this paper: One can determine the stability properties more detailed. The closer the reflection factor is to zero the more robust the impedance $Z^{*}$, i.e. the telepresence system with force feedback, is against instabilities.

\subsection{Impact on Transparency}

The impedance $Z^{*}$ encountered by the human operator handling the HSI is given as

$$
Z^{*}(s)=\frac{F_{h s i}(s)}{s x_{h s i}(s)}=b-2 b \frac{e^{-2 s T} R(s)}{1+e^{-2 s T} R(s)} .
$$

In case of an impedance matched system, $Z^{*}=b$, at the end of the communication line the reflection factor $R$ is zero and consequently the right addend of equation (18) disappears. Hence, impedance matching over all frequencies does only occur if the remote environment equals a pure damper of the magnitude $b$. If the reflection factor is not zero the right addend of equation (18) does not disappear providing a signal which depends on the time delay and on the reflection factor. Investigating the limits for the frequencies $s=0$ and $s \rightarrow \infty$ yields

$$
Z^{*}(0)=Z_{x}(0), \quad \lim _{s \rightarrow \infty} Z^{*}(s)=b .
$$

This means that only at very low frequencies the operator perceives approximately the environment impedance.

Equation (19) proposes the choice of the wave impedance $b$. If it is set to

$$
b:=\lim _{s \rightarrow \infty} Z_{x}(s)
$$


then transparency, $Z^{*}=Z_{x}$, is at least nearly reached for very low and very high frequencies. Approximating the time delay by

$$
e^{-2 s T} \approx \frac{1}{1+2 T s},
$$

leads to a more applicable form of equation (18)

$$
Z^{*}(s) \approx b \frac{1+2 T s-R(s)}{1+2 T s+R(s)} .
$$

This is the 1st order approximation of the impedance that the human operator perceives. For example, if the teleoperator works in free space, $Z_{x}=0$, the operator perceives the impedance

$$
\left.Z^{*}(s)\right|_{Z_{x}=0} \approx \frac{b \cdot T s}{1+T s}=\frac{p \cdot m s}{p+m s} .
$$

This expression states that the human operator approximately perceives an impedance that consists of a damper $p=b$ and a mass $m=b T$ in serial connection if the remote environment is free space. It has to be emphasized that the mass is proportional to the time delay $T$. Hence the transparency of the system is effected by the magnitude of the delay and by the magnitude of the characteristic impedance of the passivated communication line.

External disturbances also have a severe impact on the telepresence system. General statements can be made regarding exemplarily the disturbance $V$ in Fig. 3. The disturbance transfer function with respect to the teleoperator is

$$
S_{h s i}(s)=\frac{F_{h s i}(s)}{V(s)}=\frac{(1-R(s)) b e^{-s T}}{1+e^{-2 s T} R(s)}
$$

Polynomial factorization yields

$$
\begin{array}{r}
S_{h s i}(s)=(1-R) b\left[e^{-s T}-R e^{-3 s T}+\ldots\right] \\
=(1-R) b\left(\sum_{k=0}^{\infty}(-R)^{k} \cdot e^{(-2 k-1) s T}\right)
\end{array}
$$

Latter equation unveils some characteristics of the way an impulse-shaped disturbance $V(s)=1$ is processed within the telepresence system: (a) The disturbance is periodical with the period $2 T$. (b) If $R>0$, its periodic successors alternate. If $R<0$, its periodic successors have the same signs as the disturbance itself.

\section{SIMULATIONS}

\subsection{Bode Plot}

The amplitude response of the telepresence system working in free space is shown in Fig. 5. The free space environment was set to

$$
Z_{x}=\frac{1}{s+11}
$$

and not to zero because the teleoperator dynamics provide a small dynamical influence. The delays are set to $T=0.1 \mathrm{~s}$. The impedance encountered by the human operator driving the HSI, $Z^{*}$, approaches the values calculated in (19) for very high and very low frequencies. To outline the approach of $Z^{*}$ to the wave impedance $b$ it was set to $b=50$ only for this diagram. Especially for commanded velocities with high frequencies the reflected force is disturbed by high frequency wave reflections that are hardly predictable since the amplitude changes very rapidly. Furthermore

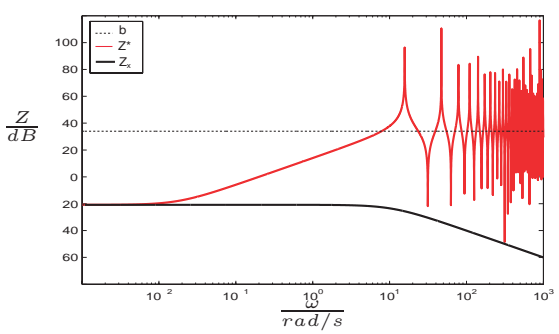

Fig. 5. Wave reflections falsify the reflected force signal

the diagram shows that the wave impedance $b$ must be adjusted with respect to the impedance $Z_{x}$ encountered by the teleoperator to obtain a telepresence system that is as transparent as possible.

\subsection{Wave Reflections by additional disturbances}

The second simulation demonstrates an impedancematched system that is disturbed shortly at $10 \mathrm{~s}$ and excited by

$$
\dot{x}_{h s i}=\sin \left(2 \frac{r a d}{s} t\right) .
$$

The time delays are again $T=0.1 \mathrm{~s}$. As it can be seen by the normalized quantities of Fig. 6 constant phase and amplitude error do not appear. However, the solitary disturbance continues with the period of the round trip delay $2 T=0.2 \mathrm{~s}$ and decreases transparency.

\subsection{Stability of a Non-Matched System}

The last simulation, displayed in Fig. 7, illustrates the dependency of the telepresence system's stability on the reflection factor $R$. It has the same configuration as in the latter simulation, but the wave impedance is $b=1$. The upper left diagram shows the reflected force $F_{h s i}$ of a telepresence system having a reflection factor that fulfills the small gain theorem (13). This can be seen in the lower left diagram. Thus wave reflections have always a fading-out behavior. If the configuration is chosen to yield a reflection factor that does not obey the small gain theorem, then wave reflection can lead to instability. In this case at least one subsystem is not passive anymore. 


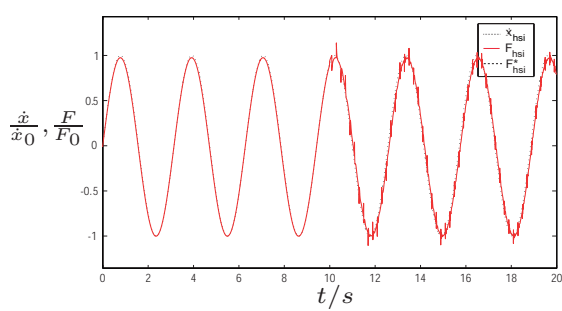

Fig. 6. Wave reflections generated by external or internal disturbances
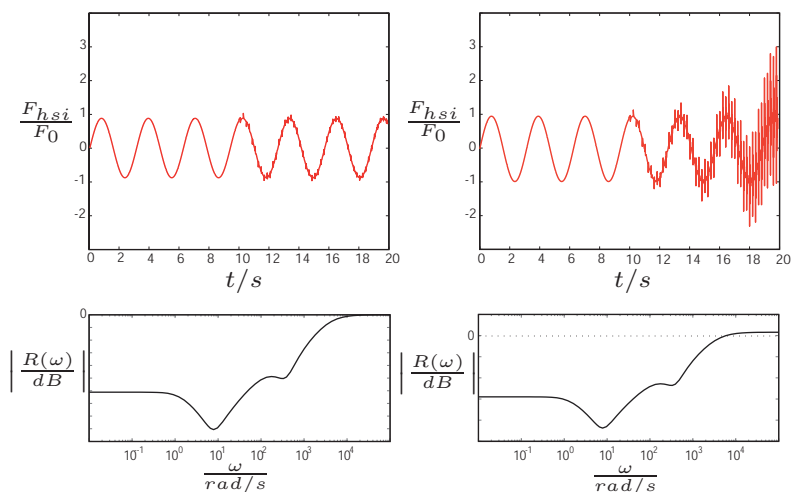

Fig. 7. Stability behavior can be characterized by the reflection factor

\section{EXPERIMENTS}

The experimental setup, Fig. 8, consists of two identical SCARA-robots working as HSI and teleoperator (TO). They are connected over a PC. For the first experiment only the teleoparator was

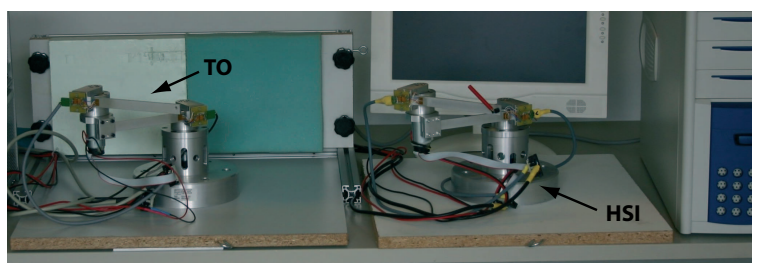

Fig. 8. Experimental setup

used and driven by the sine function (27). In the second experiment a human operator manipulated the HSI and steered the teleoperator toward two different environments. The first experiment illustrated in Fig. 9 shows the teleoperator encountering free space. The impedance $Z^{*}$ is only approximate zero since dynamics of the operator cause a small deviation. The actual remote impedance is already used in the simulation part and defined in (26). The result of the second experiment is displayed in Fig. 10. Although the reflected force gives the human operator an impression of the remote environment wave reflections cause deteriorations diminishing transparency.

\section{CONCLUSION}

In this paper it is shown that wave reflections, as arising in haptic telepresence systems with

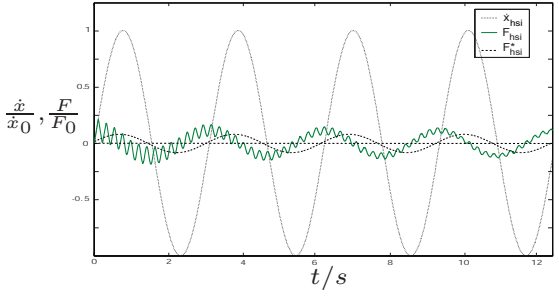

Fig. 9. Teleoperator encountering free space

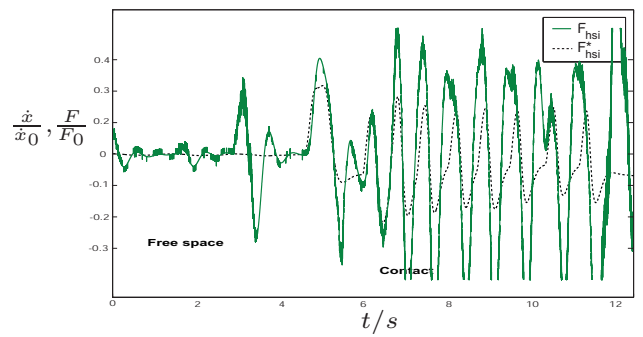

Fig. 10. Telepresence in free space and contact

passivated communication line, carry information of the reflected force signal as well as they provide deteriorations that diminish transparency.

A telepresence system, which deploys the wave transformation is not able to provide a transparent perception of a remote environment. The wave transformed communication line is not suitable to submit two signals that carry information which is partly independent. However, the wave transformation provides a stable communication line when constant time delays occur.

\section{ACKNOWLEDGEMENTS}

This work was supported by the DFG, SFB 453 . Thanks to the institute's shop for support.

\section{REFERENCES}

Anderson, R. and M. Spong (1989). Bilateral Control of Teleoperators with Time Delay. In: Proceedings of the IEEE International Conference on Robotics and Automation. -. pp. vol.34, pp. 494-501.

Hirche, S. and M. Buss (2003). Passive Position Controlled Telepresence System with Time Delay. In: Proceedings of the American Control Conference. Denver, CO.

Lawrence, D. (1993). Stability and Transparency in Bilateral Teleoperation. IEEE Transaction on Robotics and Automation 9, 624-637.

Niemeyer, G. (1996). Using Wave Variables in Time Delayed Force Reflecting Teleoperation. PhD thesis. MIT. Department of Aeronautics and Astronautics. -.

Niemeyer, G. and J. Slotine (1997). Using Wave Variables for System Analysis and Robot Control. In: IEEE Conf. on Robotics and Automation. Albuquerque, NM. pp. vol. 2, pp. 1619-1625. 